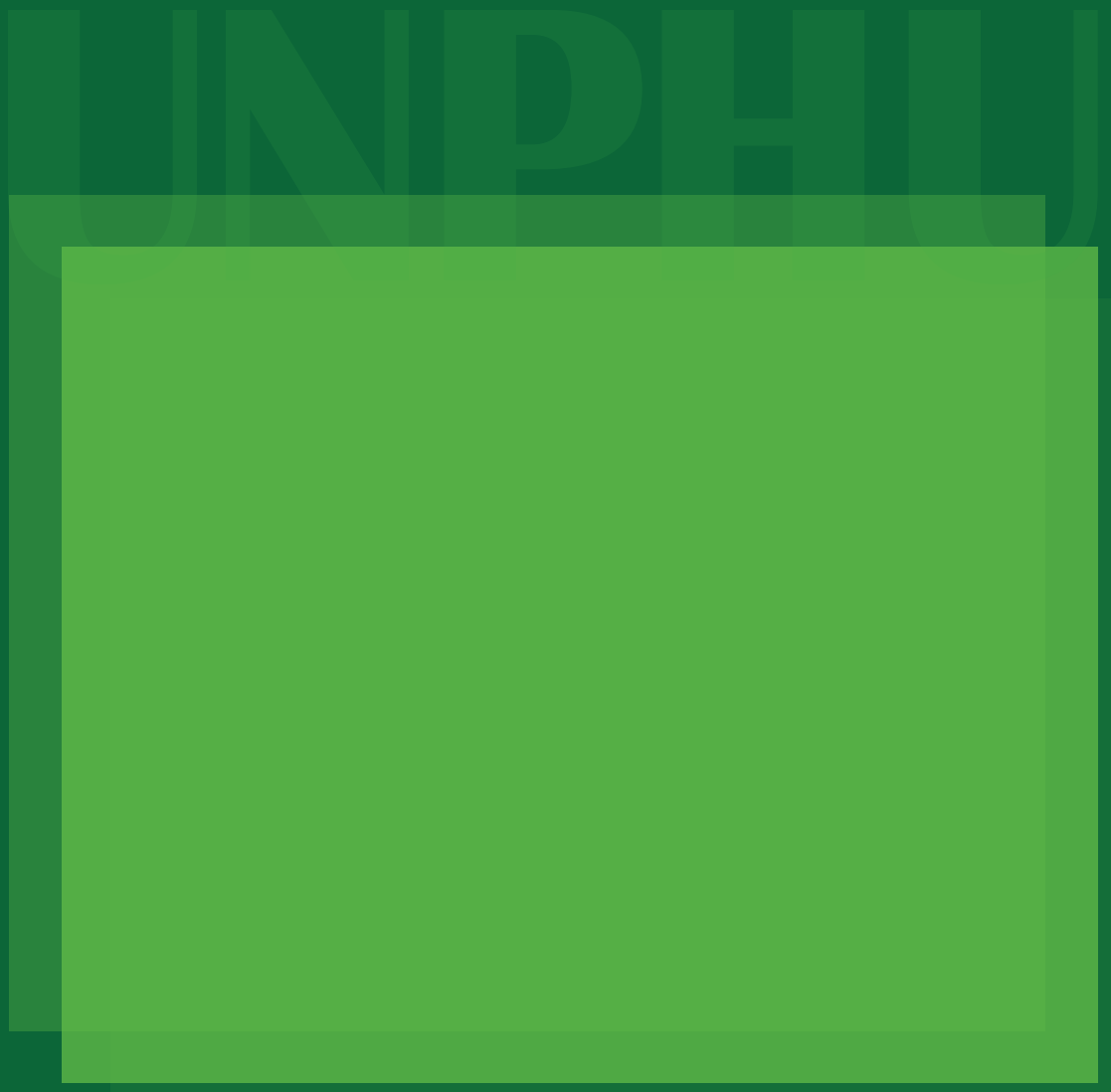




\section{Uso de hábitat de anidación del Gavilán de la Española (Buteo ridgwayi) en el Parque Nacional Los Haitises, República Dominicana.}

\section{Use of nesting habitat for the Ridgway's Hawk (Buteo ridgwayi) in Los Haitises National Park Dominican Republic.}

Eladia María Gesto De Jesús

Centro Agronómico Tropical de Investigación y Enseñanza (CATIE). Fundación Propagas.

Egesto@fundacionpropagas.do

Fecha de recepción: \# de\# de 2018.

Fecha de aceptación: \#de \# de 2018.

Favor de citar este artículo de la siguiente forma:

Gesto, E. (2018). Uso de hábitat de anidación del Gavilán de la Española (Buteo ridgwayi) en el Parque Nacional

Los Haitises, República Dominicana.

Revista AULA. Vol. 61, Número 2, enero-junio 2018. Santo Domingo: Amigo del Hogar

\section{RESUMEN}

El Gavilán de la Española (Buteo ridgwayi) es una rapaz endémica de la Española considerada el ave más amenazada del país y una de las rapaces más amenazadas del mundo, con una distribución histórica de unos 27 puntos de la Española, reduciendo su población en la actualidad a único hábitat natural, el Parque Nacional Los Haitises. La determinación de los patrones de hábitat para anidación de esta ave es importante como insumo al conocimiento de sus preferencias como población vulnerable para fines de acciones de conservación de la especie. Para la determinación de patrones de uso del hábitat del Buteo ridgwayi, se muestrearon 186 árboles para los que se tomaron variables dasométricas y de cobertura de vegetación.

Para la elección de especies de árboles de anidación del Buteo ridgwayi, las variables altura y especie fueron las de mayor relevancia para el ave. El árbol de anidación de preferencia fue la Roystonea borinquena con un $81 \%$ de los casos. El uso de suelo con mayor presencia de nidos fue el matorral con un 43\% de los casos. Los nidos del Buteo ridgwayi presentaron valores inferiores en la métrica distancia mínima del vecino más cercano en comparación con otros registros para especies del género Buteo. La proximidad entre nidos es un indicativo de la escasez de condiciones necesarias en el entorno actual para la población bajo monitoreo. Los nidos del Buteo ridgwayi estuvieron presentes en diferentes usos de suelo en zonas fragmentadas y deforestadas, por lo que no se muestra una dependencia directa del bosque para efectos de anidación.

Palabras clave: Gavilán de la Española, Buteo ridgwayi, hábitat, aves endémicas, Parque Nacional, anidación 


\begin{abstract}
The Ridgway's Hawk (Buteo ridgwayi) is an endemic raptor to the Hispaniola, considered the most threatened bird in Dominican Republic and one of the most endangered raptors of the world, with an historical distribution of 27 spots in the island reduced its population to one only remaining habitat Los Haitises National Park. The establishment of nesting habitat patterns for this bird is an important data for the understanding of its environmental preferences as vulnerable population, in order to be useful for the decision taking in conservation actions for the specie. For determining the habitat use patterns of the Buteo ridgwayi, 186 trees were sampled. Measures of dasometric variables and vegetation coverture were taken. For the estimation of landscape patterns in a fragmented and deforested environment, landscape metrics in a $250 \mathrm{mt}$ of buffer area and the home range area was calculated.
\end{abstract}

For the selection of the nesting tree species by the Buteo ridgwayi, the variables height and spp presented the higher relevance for the bird. The nesting tree of preference was Roystonea borinquena with $81 \%$ of the nesting cases. The land use Scrubland had the majority of the cases of nesting with $43 \%$ of the cases. The nests of Buteo ridgwayi had lower values for the metric nearest neighbor distance in comparison with other species of the genus Buteo. The proximity in distance between nests indicates of the scarcity of adequate conditions in the environment of this population under monitoring. The nest of Buteo ridgwayi were located in different land uses in fragmented and deforested areas showing there is not a direct dependence with the forest for nesting.

Keywords: Ridgway's hawk of Hispaniola, Buteo ridgwayi, habitat, endemic birds, National Park, nesting

Uso de hábitat de anidación del Gavilán de la Española (Buteo ridgwayi)

en el Parque Nacional Los Haitises, República Dominicana.

\section{Introducción}

Las rapaces son aves depredadoras que incluyen al grupo de los halcones, lechuzas, búhos, águilas y gavilanes. son consideradas en muchas ocasiones como especies indicadoras, debido a su posición en el tope de la cadena trófica y por su necesidad de amplitud de hábitat (Rodríguez-Estrella et al. 1998). Las rapaces tienen patrones territoriales de reocupación de áreas elegidas como lugares de anidación (Newton 1979) y por esto brindan información como especies en el estudio de características de hábitat requeridos. Además, debido a su movilidad tienen acceso a diversos parches de hábitat en el bosque con la posibilidad de evaluar la calidad de dicho hábitat según sus requerimientos (Kruger 2002). Debido a la tendencia de reocupar un lugar por más de una temporada, la elección de dichos sitios, se presume como un proceso de importancia para la especie por la influencia que este puede tener en su éxito reproductivo (Bielanski 2006).

La presencia del Buteo ridgwayi, rapaz endémica de la isla La Española, fue reportada en 1981 para República Dominicana y Haití en 27 puntos diferentes que incluyen hábitats que van desde el bosque húmedo subtropical en terrenos de formación cársica, subtropical seco, bosque de coníferas, hasta terrenos de cultivos agrícolas (Wiley y Wiley 1981). La distribución actual del Buteo ridgwayi se restringe a un solo punto en el territorio dominicano, el Parque Nacional Los Haitises (Perdomo et al. 2010); lo que convierte a su población en pequeña, fragmentada (WildEarth Guardians 2011) y vulnerable. Por esta condición se le asigna la categoría de amenaza y como especie en peligro crítico, según la lista roja de la UICN (2010). La reducción 
de hábitat en un área puede llevar al declive de la población de una especie, debido a la disminución de un espacio adecuado para su territorio, anidación, entre otros requerimientos (Rolstad 1991). La identificación de patrones que influencian la elección de un hábitat parte de una especie, brinda información acerca de qué requerimientos son adecuados para su supervivencia, aspecto crítico para un manejo efectivo en planes de conservación de especies (Chalfoun 2007).

La presente investigación evalúa las características de hábitat asociadas a lugares de anidación de la población monitoreada del Buteo ridgwayi que determinan patrones en la selección de árboles y uso de hábitat circundante para los nidos en la temporada de monitoreo 2015. Adicionalmente se describen características poblacionales del gavilán durante el período 2005 - 2015. Finalmente, esta investigación contribuye a aumentar el conocimiento del comportamiento de esta especie en las actuales condiciones de fragmentación de Los Haitises y provee una línea de base sobre sus patrones de anidación, como parte de los esfuerzos para la reconstrucción de la historia natural de esta especie endémica amenazada y para su plan de conservación.

\subsection{Descripción del área de estudio}

El Parque Nacional Los Haitises (PNLH) se encuentra en la zona nordeste de la República Dominicana. Fue creado en 1976 y en la actualidad tiene una extensión de $631 \mathrm{~km}^{2}$ (MIMARENA 2013a). Los Haitises se ubica en la zona que registra mayor cantidad de lluvias para todo el país, con una precipitación anual de alrededor de 2,000 milímetros (Comisión 2014) y donde están representadas las zonas de vida bosque muy húmedo y húmedo tropical, bosque de mangles, zonas húmedas, formaciones de roca karst (de roca caliza) y línea costera (Jaragua 2009). Su formación geológica es caracterizada por la formación de mogotes o pequeñas colinas, que son una serie de elevaciones próximas unas a otras y con fondos de por medio, de 40 a $380 \mathrm{msnm}$ (Mateo 2000). Como área natural protegida, Los Haitises son una de las 27 áreas importantes para la conservación de las aves (IBA), y una de las áreas claves para la biodiversidad (KBA) del país (Jaragua 2009) cuyos criterios de elegibilidad son: ser hábitat para especies globalmente amenazadas (Criterio de Amenaza), de distribución restringida y con congregaciones importantes (Criterio de Irremplazabilidad).

El Parque Nacional Los Haitises constituye el único refugio natural de hábitat del Buteo ridgwayi. Para fines de conservación de la especie, bajo el proyecto "Restauración de la Distribución de Abundancia del Gavilán de La Hispaniola en República Dominicana" se realizó una exploración en caminata en todo el Parque Nacional Los Haitises y se ha identificado la presencia del ave en diversos puntos del parque. Luego de la exploración, realizada por el personal de The Peregrine Fund, se seleccionaron tres zonas para el monitoreo de nidos del Buteo ridgwayi: Los Limones, Cristal y Sabana de la Mar. Estas zonas fueron escogidas por presentar menor grado de dificultad de acceso por vía terrestre en comparación con otras áreas en el parque, cuya topografía y puntos de presencia de nidos dificultan la realización de un programa de monitoreo constante. El proyecto inició en el año 2002 y tiene como principales tareas para aumentar el crecimiento de la población monitoreada: el anillado y monitoreo de individuos identificados a través de los años, tratamiento de polluelos afectados por la larva Philornis pici, la aplicación de la técnica de hacking (selección de polluelos, crianza en nidos artificiales y posterior liberación al medio natural) para su reintroducción en la zona de Punta Cana (en la costa este del país), y el componente de educación a poblaciones campesinas cercanas a zonas de anidación para reducir impactos por cacería. 


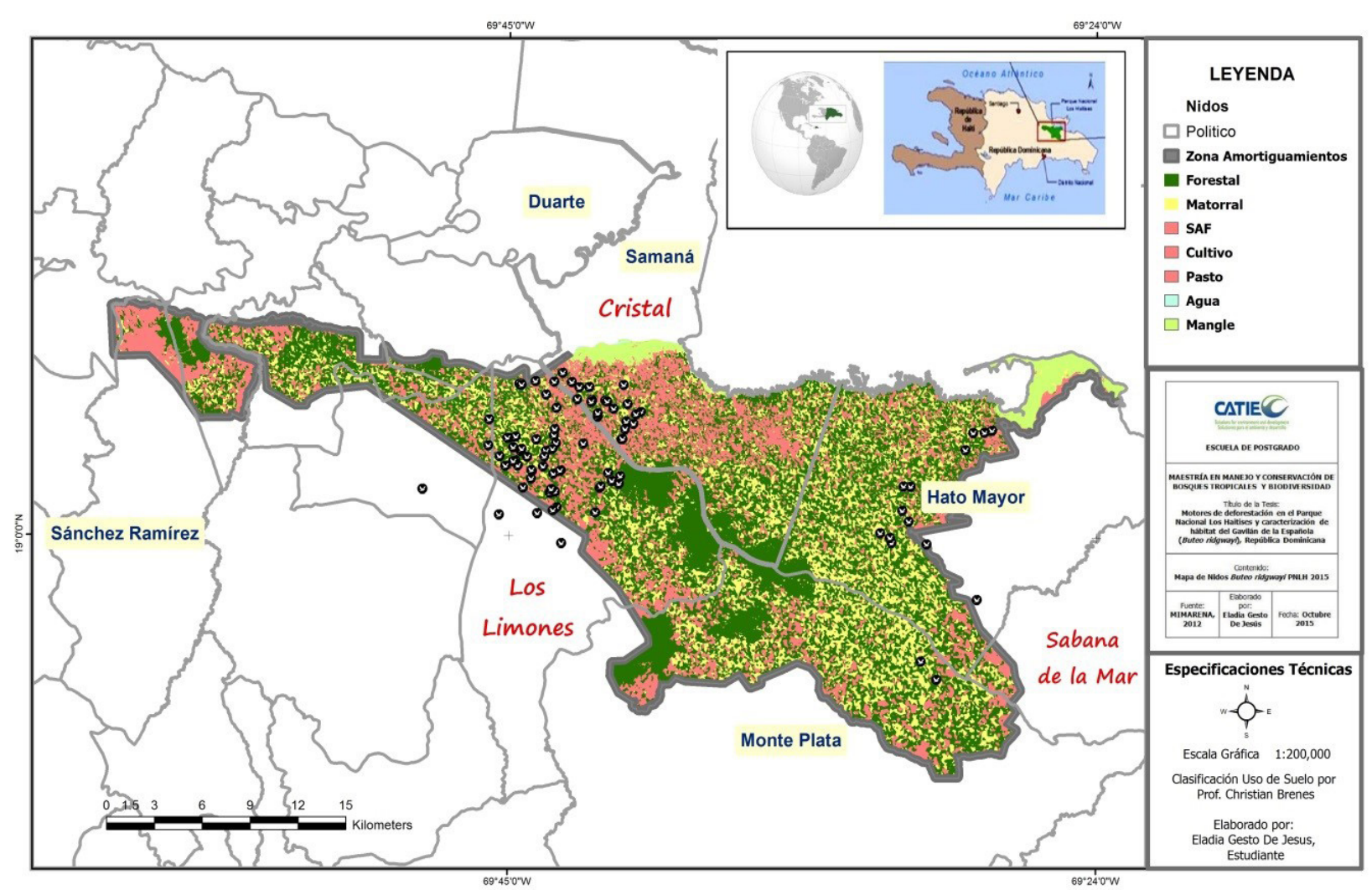

Figura 1: Mapa de uso de suelo del Parque Nacional Los Haitises y distribución de nidos monitoreados del Buteo ridgwayi (Elaboración propia).

\section{Procedimientos metodológicos}

Para la caracterización del uso de hábitat de anidación del Buteo ridgwayi en el Parque Nacional Los Haitises, se muestrearon todos los nidos activos e intentos de nidos registrados en el proyecto para la temporada 2015, que correspondieron a 93 árboles de anidación durante los meses de junio - julio en las tres zonas del proyecto.

\subsection{Muestreo de árboles de anidación y há- bitat circundante}

Se obtuvo información de dos grupos de árboles: árboles de anidación y árboles vecino. El árbol vecino fue definido para este caso como el árbol no anidado de igual especie que el árbol nido, que se encontrase a la menor distancia en un radio de $50 \mathrm{~m}$. En ausencia de estas con- diciones, se eligió el árbol más cercano al árbol nido en $50 \mathrm{~m}$ de radio que perteneciera a una de las especies resgistradas de anidación del Buteo ridgwayi. La inclusión del árbol vecino en la toma de datos permitió hacer un contraste de características respecto al árbol nido y por lo tanto buscar algún patrón de preferencia de la especie respecto a los árboles para anidar.

La muestra correspondió a 186 árboles, 93 árboles nido y 93 árboles vecino. Para cada árbol nido y vecino se recolectaron las variables indicadas en el cuadro 1.

Para la obtención de las medias de las variables, toda la información fue posteriormente tabulada en una base de datos de Microsoft Excel y exportada al software Infostat para su análisis estadístico. Los puntos de GPS fueron exportados al software Arc Gis 10.0 con el cual se definieron zonas de entorno sobre 
la capa de uso de suelo 2012 para el Parque Nacional Los Haitises obtenidas a partir del Mapa de Uso de Suelo 2012 de República Dominicana (MIMARENA 2014a). Finalmente, las imágenes de zonas de entorno fueron exportadas al software Fragstat 4.2 para el análisis de patrones espaciales.

Cuadro 1

Variables de estudio entre los árboles nido y vecino del Buteo ridgwayi. (Elaboración propia).

\begin{tabular}{|c|c|}
\hline Variable & Herramienta para la toma de datos \\
\hline Spp & Identificación con personal de campo \\
\hline Altura del árbol & $\begin{array}{l}\text { Altura desde la base del árbol hasta su ápice. Se usó un } \\
\text { clinómetro Suunto Pm-5/1520 a } 15 \text { metros de distancia de la } \\
\text { base del árbol }\end{array}$ \\
\hline Altura de la copa & $\begin{array}{l}\text { Medida resultante de restar a la altura del árbol, la distancia } \\
\text { que va de la base del árbol al punto más bajo de la copa del } \\
\text { árbol. Se usó un clinómetro Suunto Pm-5/1520 a } 15 \text { metros } \\
\text { de distancia de la base del árbol }\end{array}$ \\
\hline Altura del nido & $\begin{array}{l}\text { Clinómetro Suunto Pm-5/1520 a } 15 \text { metros de distancia de } \\
\text { la base del árbol }\end{array}$ \\
\hline Ramas u hojas & $\begin{array}{l}\text { Conteo visual de ramas u hojas principales que salen del } \\
\text { tronco del árbol o palma }\end{array}$ \\
\hline Diámetro a la altura & $\begin{array}{l}\text { Cinta diamétrica con datos tomados a } 130 \mathrm{~cm} \text { sobre la base } \\
\text { del árbol }\end{array}$ \\
\hline \multirow{2}{*}{$\begin{array}{l}\text { Cobertura de vegetación dentro } \\
\text { de una radio de } 5 \mathrm{~m}\end{array}$} & Densiómetro esférico a una distancia de $5 \mathrm{~m}$, desde la base \\
\hline & $\begin{array}{l}\text { Escala de Thiollay (WWF 2004) a una distancia de } 5 \mathrm{~m} \text { de la } \\
\text { base del árbol para cada punto cardinal }\end{array}$ \\
\hline \multicolumn{2}{|c|}{$\begin{array}{l}\text { Estructura vertical dentro de un } \\
\text { radio de } 5 \mathrm{~m}\end{array}$} \\
\hline $\begin{array}{l}\text { Pendiente de inclinación del } \\
\text { árbol }\end{array}$ & $\begin{array}{l}\text { Uso del clinómetro Suunto Tandem-360PC/360R a } 15 \\
\text { metros de distancia en dirección norte desde la base del árbol } \\
\text { para la estimación de la pendiente }\end{array}$ \\
\hline \multirow{2}{*}{ Coordenadas y altitud (msnm) } & $\begin{array}{l}\text { Uso del GPS Garmin Extrex 20, se toman los datos cuando } \\
\text { el equipo indicó un error mínimo de } 3 \mathrm{~m}\end{array}$ \\
\hline & $\begin{array}{l}\text { Reconocimiento visual en campo de vegetación dominante } \\
\text { en un radio de } 50 \mathrm{~m}\end{array}$ \\
\hline
\end{tabular}


Cuadro 2

Descripción de las coberturas de suelo presentes en el estudio según categorías del Ministerio de Medio Ambiente y Recursos Naturales. Fuente: MIMARENA (2014c).

\begin{tabular}{ll}
\hline Cobertura & \multicolumn{1}{c}{ Descripción } \\
\hline Cultivo & $\begin{array}{l}\text { Cultivos perennes, anuales o de ciclo corto, donde predominan los } \\
\text { tubérculos }\end{array}$ \\
Forestal & Bosque latifoliado húmedo, perennifolio en elevaciones entre 500 y \\
& $2,000 \mathrm{msnm}$, con un rango pluviométrico de 1,500 a $2,000 \mathrm{~mm}$ \\
Matorral & Especies arbustivas y especies arbóreas que crecen en áreas que están \\
en proceso de regeneración natural de una altura máxima de 5 metros & Suelos de pastizal natural e intensivo destinado a la actividad ganadera \\
\hline
\end{tabular}

\subsection{Análisis de datos}

Variables dasométricas y de cobertura de vegetación

Con el fin de verificar posibles diferencias o no entre árboles nido y vecino, que expliquen tendencias importantes en las características de los árboles, con el uso del software Infostat (M.G. et al. 2008) se aplicó la prueba T apareada para las medias de las variables cuantitativas. Se usaron tablas de contingencia y tablas de frecuencia para la comparación de variables categóricas.

Variables de importancia en la elección del árbol de anidación

Para determinar qué variable o variables mostraron una relación significativa con la presencia de árboles elegidos para anidación por el Buteo ridgwayi, se realizó una regresión logística. Una vez identificadas las variables con valor $\mathrm{p}>0.05$, se aplicó con ellas la herramienta: modelos lineales generales mixtos (MLGM). Se usó como variable dependiente la variable nido y como criterios de clasificación las variables categóricas.
De igual manera, respecto a la muestra de árboles nido, se realizó una regresión logística teniendo como variable dependiente el número de nido. Posteriormente, se aplicaron pruebas de modelos lineales generalizados con la herramienta de prueba de hipótesis secuenciales para efectos fijos; teniendo como criterios de clasificación las variables categóricas nido, zona de ubicación y especie.

\section{Uso de suelo y métricas de paisaje}

Para la descripción de características en el hábitat circundante de los nidos del Buteo ridgwayi , se usaron métricas de clase (referentes a la cobertura de suelo) y métricas de paisaje (Turner 1989). El cuadro 2 indica los tipos de coberturas presentes en la zona de estudio:

A partir de los puntos GPS de los 93 árboles nidos, se obtuvieron mediante el software Arc Gis áreas de entorno de $250 \mathrm{~m}$ de radio teniendo como punto central el árbol nido, sobre las capas de uso de suelo para el Parque Nacional Los Haitises (MIMARENA 2014a) y se generaron 93 imágenes de áreas de entorno. 
Cuadro 3

Descripción de métricas de paisaje según definiciones de McGarigal (2015). (Elaboración propia)

\begin{tabular}{lccl}
\hline \multicolumn{1}{c}{ Métrica } & Abreviatura & Unidad & \multicolumn{1}{c}{ Descripción } \\
\hline $\begin{array}{l}\text { Tamaño } \\
\text { de parche }\end{array}$ & AREA & Ha & $\begin{array}{l}\text { Área de cada unidad de parche dentro de la unidad } \\
\text { de paisaje }\end{array}$ \\
$\begin{array}{l}\text { Número } \\
\text { de parches }\end{array}$ & NP & $\#$ & $\begin{array}{l}\text { Cantidad de fragmentos por unidad de paisaje. A } \\
\text { mayor cantidad de parches, mayor fragmentación }\end{array}$ \\
$\begin{array}{l}\text { Porcentaje } \\
\text { del paisaje }\end{array}$ & PLAND & $\%$ & $\begin{array}{l}\text { Porcentaje ocupado por un tipo de cobertura en } \\
\text { una unidad de paisaje. A mayor valor, mayor pres- } \\
\text { encia en la cobertura }\end{array}$ \\
$\begin{array}{l}\text { Índice del } \\
\text { parche mayor }\end{array}$ & LPI $\%$ & $\begin{array}{l}\text { Porcentaje que ocupa el parche más grande por } \\
\text { unidad de paisaje. A mayor valor, menor frag- } \\
\text { mentación }\end{array}$ \\
\hline
\end{tabular}

Posteriormente, se exportaron las 93 imágenes al software Fragstat 4.2 para el análisis de patrones espaciales. Se obtuvieron métricas de paisaje, considerando cada una de las 93 áreas de entorno como unidades de paisaje, de $250 \mathrm{~m}$ de radio cada una. Las métricas de clase y paisaje consideradas para este estudio se describen a continuación. Las métricas correspondieron a datos descriptivos y no a pruebas estadísticas debido al solapamiento entre las zonas de entorno. Se muestran a continuación en el cuadro 3 .

Los resultados de métricas para las 93 unidades de paisaje fueron arreglados en Microsoft Excel y posteriormente importados a Infostat para la obtención de las medias de cada métrica.

Cálculo de la distancia del vecino más cercano (MEAN_ENN) y el rango de hogar (Home Range)

Las áreas de entorno por nido fueron consideradas como unidades individuales de paisaje. Para el cálculo de la media de la distancia Euclidia al nido más cercano (MEAN_ENN), se utilizó el software Arc Gis. El ENN se calculó de forma global para todas las unidades de áreas de entorno de árboles y posteriormente se obtuvo con el uso de Infostat, las medias para todo el parque, así como también para las zonas de Los Limones, Sabana de la Mar y Cristal. Estos datos fueron comparados con estimaciones previas a este estudio, en investigaciones de 1981 (Wiley y Wiley 1981) y del 2005 al 2009 (Woolaver 2011) para la identificación de cambios en el tamaño del territorio del ave a través del tiempo. En la figura 2 se muestra un gráfica de cómo se visualizan estos cálculos.

Se calculó el rango de hogar teniendo en cuenta que su concepto está restringido en este estudio al área de anidación. Para el cálculo del rango de hogar del Buteo ridgwayi, se usó la fórmula del área de una circunferencia con una modificación: $\mathrm{A}=\prod \mathrm{r}^{2} * 1.158$, donde $\mathrm{r}$ es la media obtenida de la distancia del vecino más cercano (ENN) y 1.158 es una constante que representa una porción de área de no traslape entre territorios de nidos distintos (Brown 1975). Esta fórmula de cálculo para rangos de hogar fue usada en el estudio de Woolaver (2011) sobre el Buteo ridgwayi y además en un estudio realizado por The Peregrine Fund, sobre densidad de nidos de una rapaz en Centroamérica (De J. Vargas González y Vargas 2011). 


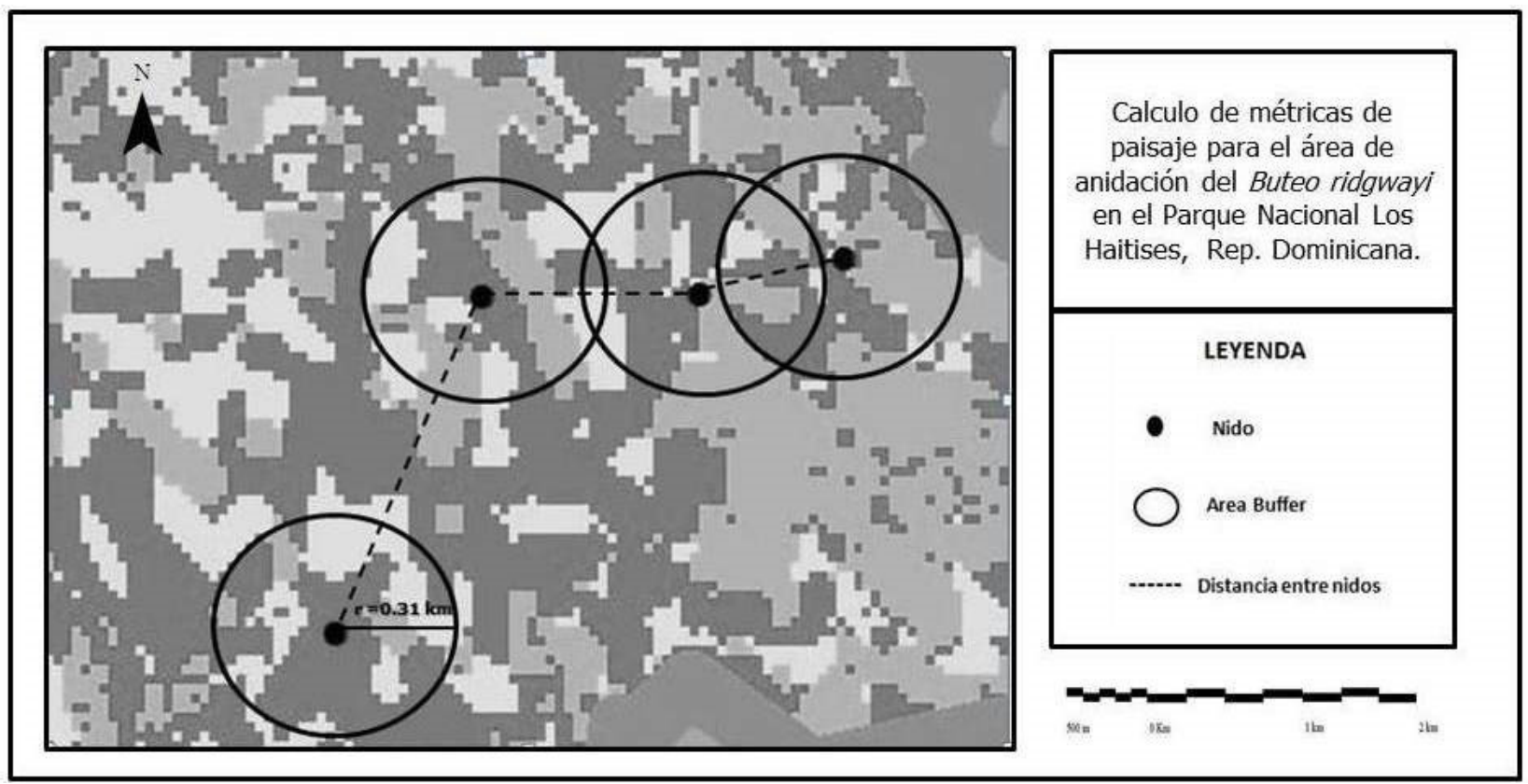

Figura 2: Diagrama explicativo del cálculo de métricas MEAN_ENN y rango de hogar para el área de anidación del Buteo ridgwayi, Parque Nacional Los Haitises, República Dominicana (Elaboración propia).

Cuadro 4

Prueba T apareada para variables numéricas de árboles nido y vecino para el Buteo ridgwayi en el Parque Nacional Los Haitises, República Dominicana (Elaboración propia).

\begin{tabular}{lccccccc}
\hline Variable & $\begin{array}{c}\text { Media } \\
(\text { dif })\end{array}$ & $\begin{array}{c}\text { Media 1 } \\
\text { (Nido) }\end{array}$ & $\begin{array}{c}\text { Media 2 } \\
\text { (Vecino) }\end{array}$ & Intervalos & D.E. & P & N \\
\hline Ramas & 2.48 & 15.9 & 13.42 & $1.26-3.70$ & 5.92 & 0.0001 & 93 \\
Altura del árbol & 3.47 & 19.89 & 16.42 & $2.18-4.76$ & 6.27 & $<0.0001$ & 93 \\
Altura Copa & 1.32 & 11.52 & 10.2 & $0.11-2.53$ & 5.87 & 0.032 & 93 \\
DAP & 15.8 & 48.89 & 33.1 & $5.36-26.23$ & 50.68 & 0.0034 & 93 \\
Vegetación & 0.12 & 0.31 & 0.19 & $0.02-0.22$ & 0.49 & 0.02 & 93 \\
$20-30 \mathrm{~m}$ & & & & & & & \\
\hline
\end{tabular}

\section{Resultados y discusión}

\subsection{Diferencias entre árboles nido y vecino más cercano}

Para el análisis de T-apareada en el total de las muestras de árboles nido y vecino, se observaron diferencias significativas en las variables ramas, altura del árbol, altura de la copa, diámetro a la altura del pecho (DAP) y vegetación de $20-30 \mathrm{~m}$ como se muestra en el cuadro 4 .

La preferencia del género Buteo en la selección de especies de árboles, cuyas medidas en las variables altura del árbol, altura de la copa y diámetro a la altura del pecho (DAP) 
sean mayores que otras especies de árboles, ha sido reportada por Virani (1999) para el Buteo augur y por Bielanski (2006) para el Buteo buteo. En el caso del Buteo ridgwayi, para árboles de la misma especie o entre especies reconocidas como árboles de anidación presentes en los lugares de anidación, el ave mostró la tendencia de ocupar un árbol de mayor altura.

Cuadro 5

Prueba de hipótesis para las variables de importancia en la selección de nidos del Buteo ridgwayi en el Parque Nacional Los Haitises, República Dominicana (Elaboración propia).

\begin{tabular}{llllll}
\hline & Df & Deviance Resid. & Df & Resid. Dev & Pr (>Chi) \\
\hline Spp & 13 & 34.63 & 172 & 223.22 & 0.0010 \\
Ramas & 26 & 30.14 & 146 & 193.08 & 0.2617 \\
Altura del árbol & 1 & 13.76 & 145 & 179.32 & 0.002 \\
Altura de la copa & 1 & 0.78 & 144 & 178.55 & 0.3774 \\
V_5_10_m & 1 & 0.70 & 143 & 177.84 & 0.4022 \\
V_20_30_m & 1 & 1.00 & 142 & 176.85 & 0.3184 \\
\hline
\end{tabular}

\subsection{Variables de importancia en la elección del árbol de anidación}

A partir del MLGM, se determinó que las variables de mayor relevancia para la selección de nidos por el Buteo ridgwayi fueron: altura del árbol y la especie; las especies de mayor ocurrencia de nidos según altura promediada por los árboles nido fueron Clusia minor, Cocus nucifera, Ceiba petandra y Roystonea borinquena, según se detalla en el cuadro 5 .

\section{5. Árbol de anidación y uso de suelo}

En la figura 3 se muestra una resumen de los datos resultantes de árbol de anidación y uso de suelo. La zona que presentó la mayor cantidad de nidos fue Los Limones con 46 casos, seguida por Laguna Cristal con 32 y Sabana de la Mar con 15. Se registraron 11 especies diferentes de árboles de anidación.
La especie de árbol con mayor frecuencia de anidación fue la Roystonea borinquena, con un $81 \%$ de los casos. Estos resultados coinciden en el uso de esta palma endémica como árbol de anidación del Buteo ridgwayi con el reporte de Woolaver (2011) para la zona de Los Limones en 2008 y 2009 con un $78 \%$ de casos de anidación en Roystonea borinquena y con el registro más antiguo de anidación que data de 1934 (Wiley y Wiley 1981).

Las demás especies de árboles de anidación (Cocus nucifera, Ceiba petandra y Clusia minor) que cumplen con los requisitos de altura son reportadas escasamente en la preferencia de nidos solo presentes en condiciones de fragmentos de bosque de cobertura más densa (Ceiba petandra y Clusia minor) o en cultivos (Cocus nucifera). En cuanto al uso de suelo, se encontraron 5 categorías indistintamente 


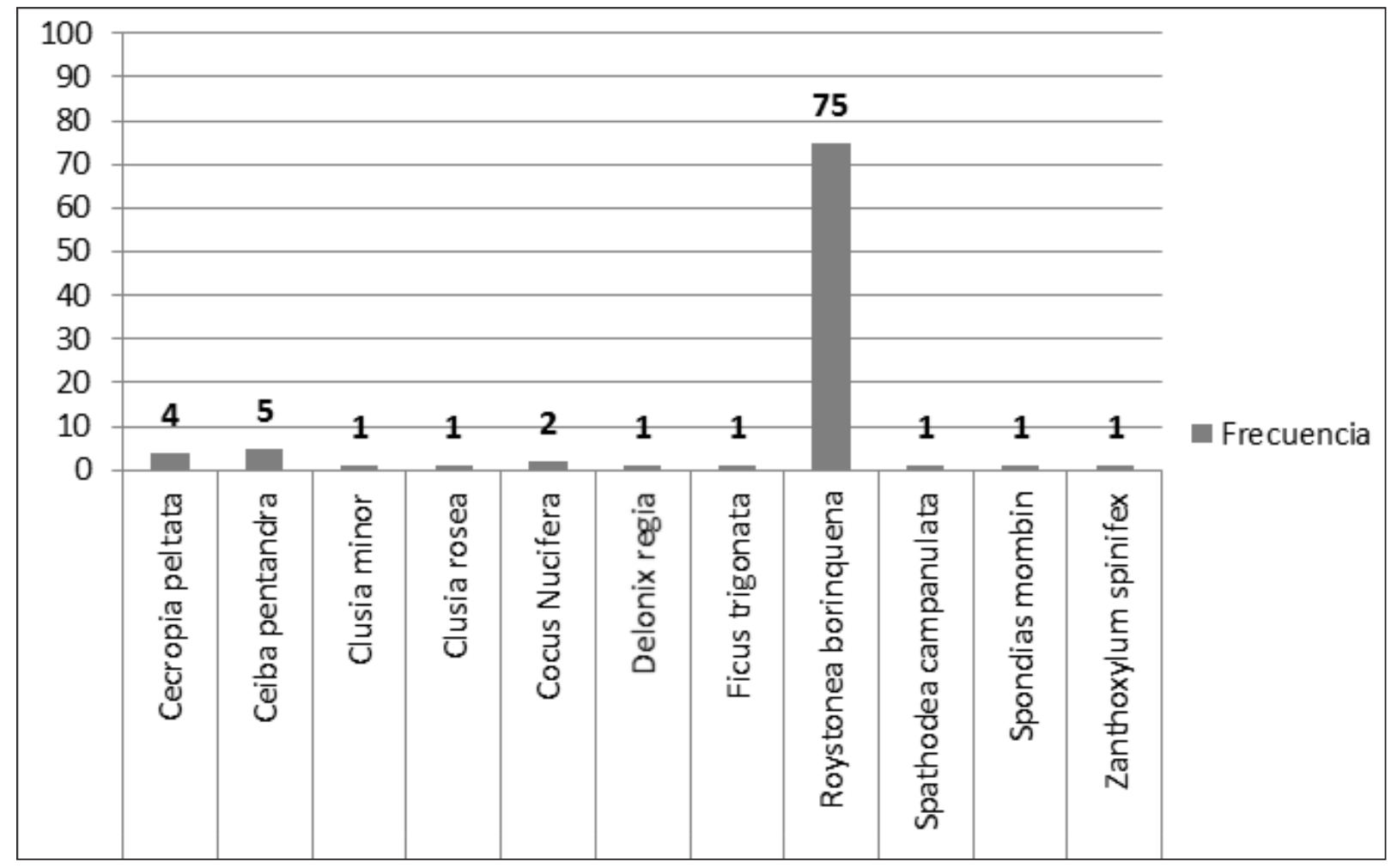

Figura 3: Frecuencia de Spp en árboles de anidación del Buteo ridgwayi en el Parque Nacional Los Haitises, República Dominicana.

de la zona; el matorral fue el uso de suelo con mayor presencia de nidos con un $43 \%$ de los casos. La preferencia del Buteo ridgwayi por la Roystonea borinquena como árbol de anidación, con registros confirmados en distintos períodos del monitoreo de la especie, responde según Woolaver (2011) a que la estructura de esta palma provee protección para condiciones ambientales, amenaza de depredadores y una estructura de anidación estable. De igual manera, la presencia de esta palma como el árbol de mayor altura sobre vegetación temprana o inexistente en los usos de suelo donde está presente en Los Haitises, se explica por dos factores: una vez el bosque es talado para dar paso a cultivos o ganadería, la palma en muchos de los casos observados es la única planta de altura que permanece por ser apreciada por los campesinos de la zona para el aprovechamiento de su madera. Según cuentan los pobladores de Los Haitises, hace unas 3 décadas, la palma fue haciéndose más visible en las zonas abiertas de Los Haitises. Por otro lado, esta palma es el árbol de anidación y fuente de alimento de otra ave endémica de República Dominicana, Dulus dominicus, por lo que la presencia de esta ave puede influir en la diseminación de la palma en el territorio como vector de dispersión. Todas las especies de árboles donde se registraron nidos para la temporada 2015 comparten una misma característica en los lugares de anidación donde se hallaban: son árboles sobresalientes en su entorno, sin competencia de dosel.

Todos los casos de nidos de Roystonea borinquena observados en campo, así como también de las demás especies de árboles nido, poseían un mismo patrón: se situaban en sitios claros con vegetación emergente (matorral) o inexistente (cultivo y pasto) en proximidad a fragmentos de bosque secundario. La selección 
del árbol nido en este tipo de configuración espacial provee algunas ventajas a la especie: permite contar con un lugar de mayor visibilidad para los fines de caza y protección de depredadores para el nido, como lo describe Virani (1999) para el caso del Buteo augur; de igual manera, la ubicación de estos árboles sobresalientes en entornos degradados del bosque de Los Haitises es de más fácil identificación en un terreno con claros de vegetación que en un fragmento de bosque denso. Esto convierte a los árboles de anidación en árboles aislados. Según Newton (1979), las rapaces presentan preferencia por árboles aislados para fines de anidación y se puede encontrar un caso similar para otra especie del género Buteo reportado por Inselman (2015) sobre la preferencia de árboles aislados para fines de anidación en un entorno de pastizales.

\subsection{Aspectos descriptivos: uso del suelo y métricas de paisaje}

Según Virani (1999) las modificaciones en el hábitat de aves rapaces pueden influenciar tanto su comportamiento predador como su patrón de anidación. A través de las métricas de paisaje, se pueden cuantificar los patrones de espaciamiento de anidación que contribuyan a la comprensión del comportamiento de la especie en un hábitat modificado. Los valores obtenidos para las métricas de paisaje, se muestran a continuación. Se obtuvieron las medias para todo el parque y además se clasificaron por uso, zona y la combinación uso y zona.

La media para la métrica tamaño de parches (AREA) correspondió a 6 ha, es decir, que el tamaño de un fragmento corresponde a $30 \%$ del tamaño de la unidad de paisaje, que tiene un área de 19 ha o $250 \mathrm{~m}$ de radio. La media del número de parches (NP) para todo el parque es de 10.16 , pero se muestran diferencias marcadas cuando se observa esta métrica según zona, siendo la zona de Cristal la más fragmentada con un valor de 15.88; mientras que para Los
Limones y Sabana de la Mar es de 7 parches. La media del índice de parche mayor (LPI) ocupa menos del 50\% del área total, tal como se muestra en el cuadro 6.

Para la métrica de porcentaje del paisaje (PLAND) relativa a la proporción de espacio que un uso de suelo ocupa en las unidades de paisaje (zonas de entorno), el uso forestal reportó una cobertura de $43.2 \%$. tal como se muestra en el cuadro 7. Es decir que, en las unidades de paisaje, el uso forestal domina en cuanto a presencia frente a otros usos. Cristal es la zona que tiene una menor proporción de cobertura de bosque en las unidades de paisaje, dato que se alinea con la métrica número de parches, donde Cristal duplica su cifra frente a otras zonas del parque. Sabana de la Mar obtiene un valor de $49.57 \%$ en su media, correspondiendo a la parte más conservada del parque. Es decir, que los nidos del Buteo ridgwayi en un radio de $250 \mathrm{~m}$ se sitúan en una configuración espacial donde el bosque no llega a ocupar un 50\% del fragmento.

Para la métrica índice del parche más largo (LPI) que se muestra en el cuadro 8, la zona que obtuvo los valores más altos fue la zona de Los Limones con un $55.56 \%$, en contraste con Cristal con el menor valor de LPI en las zonas estudiadas en el parque según se muestra en el siguiente cuadro. El valor de LPI más alto para todas las zonas de entorno en el parque se encuentra en la zona más deforestada; por lo que un LPI de mayor valor en esta sección del parque provee información sobre el avance de la deforestación a una escala de $250 \mathrm{~m}$ de diámetro. En los valores de LPI según uso y zona, se puede observar que en la zona de Los Limones se encuentra el mayor valor del LPI con un $66.58 \%$ para el uso cultivo. En Cristal, aunque con un valor menor, el cultivo también domina la media del índice. Solo el caso de Sabana de la Mar muestra diferencias respecto a las otras dos zonas: el bosque posee su mayor valor de media del LPI, seguido por el matorral con valores muy similares. 
Cuadro 6.

Métricas de clase y paisaje para lugares de anidación del Buteo ridgwayi, Parque Nacional Los Haitises, República Dominicana. (Elaboración propia)

\begin{tabular}{|c|c|c|c|c|c|c|}
\hline Variable & $\mathrm{N}$ & Unidad & Media & D.E. & Mín & Máx \\
\hline AREA & & 304 & ha & 6 & 0.09 & 19.8 \\
\hline NP & & 93 & & 10.16 & 1 & 24 \\
\hline LPI & & 93 & $\%$ & 47.43 & 10.5 & 100 \\
\hline
\end{tabular}

Cuadro 7

PLAND para lugares de anidación del Buteo ridgwayi, Parque Nacional Los Haitises, República Dominicana (Elaboración propia).

\begin{tabular}{lllllll}
\hline Pland & Uso & N & Media & D.E. & Mín & Máx \\
\hline PNLH & Cultivo & 87 & 26.67 & 20.73 & 0.91 & 98.6 \\
& Forestal & 92 & 43.2 & 15.71 & 1.4 & 89.77 \\
& Matorral & 90 & 25.13 & 15.66 & 0.47 & 61.93 \\
& Pasto & 35 & 21.23 & 17.5 & 0.46 & 100 \\
\hline
\end{tabular}

Cuadro 8

LPI según zona para lugares de anidación del Buteo ridgwayi, Parque Nacional Los Haitises, República Dominicana (Elaboración propia).

\begin{tabular}{lrcccc}
\hline LPI según zona & $\mathrm{N}$ & Media & D.E. & Mín & Máx \\
\hline Cristal & 32 & 34.43 & 12.84 & 10.50 & 58.72 \\
Los Limones & 46 & 55.56 & 17.97 & 25.57 & 100.00 \\
Sabana de la Mar & 15 & 52.09 & 14.17 & 28.57 & 75.69 \\
\hline
\end{tabular}




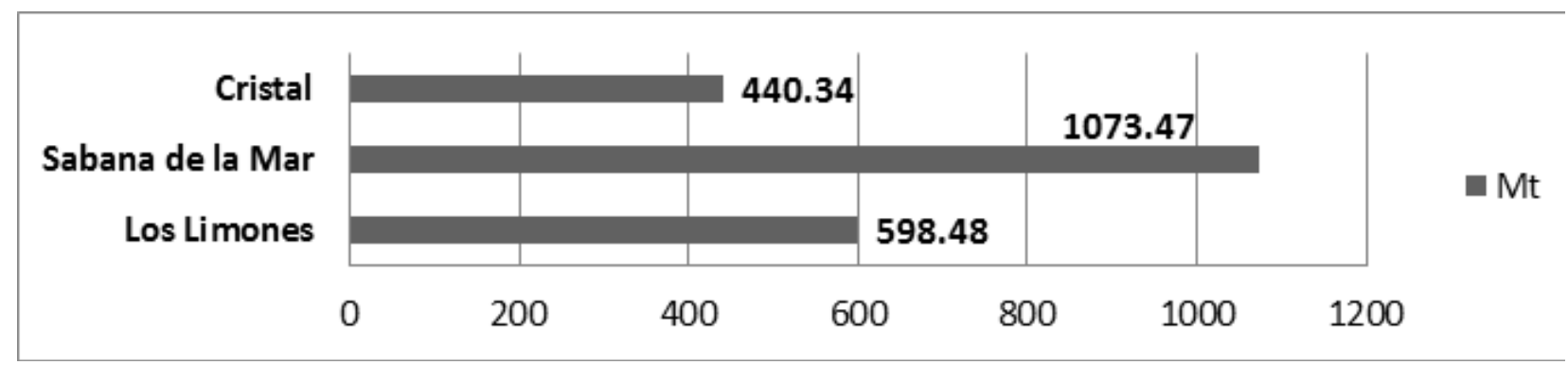

Figura 4: Valor MEAN_ENN según zona para lugares de anidación del Buteo ridgwayi, Parque Nacional Los Haitises, República Dominicana (Elaboración propia).

2.7. Distancia del vecino más cercano (MEAN_ENN) y rango de hogar para anidación

El grado de dispersión de nidos en el estudio de rapaces es cuantificado por la métrica distancia del vecino más cercano (MEAN_ENN) según se muestra en la figura 4. Para esta métrica se obtuvo un valor promedio para todo el parque de $620.28 \mathrm{~m}$ de distancia, siendo Sabana de la Mar la zona con una mayor distancia entre los nidos del Buteo ridgwayi con una media de 1,073.47 metros, lo que representa el doble de las otras dos zonas.

Al comparar los datos de la distancia mínima al vecino más cercano (MEAN_ENN) y el número de parches por zona, como se muestra en la figura 5 , se observa que a mayor número de parches, menor es la distancia entre nidos. Es decir, la zona más fragmentada es la que presenta también más solapamiento entre nidos.

Newton (1979) afirma sobre este tipo de patrones en rapaces, que una mayor proximidad entre nidos es un indicativo de que no existen suficientes parches adecuados para la anidación o fuente de alimento para dieta y los recursos se ven concentrados en lugares más pequeños. Un patrón de espaciamiento entre nidos más aislados es un indicativo de mayores condiciones en el hábitat para presentar nidos más dispersos entre sí. Sabana de la Mar posee el mayor espaciamiento entre nidos y este dato coincide con otras métricas tales como LPI y PLAND; además de la verificación en campo de ser la zona donde el bosque está mejor conservado. A pesar de este dato que indica una mejor calidad de hábitat en términos de presencia de bosque, el grupo de población más pequeña del monitoreo pertenece a esta zona. Una de las causas de esta menor presencia de nidos en condiciones ambientales más saludables se puede atribuir al esfuerzo de muestreo más reducido: menos claros de vegetación pueden dificultar la identificación de nidos en comparación con las otras zonas más fragmentadas (Cristal) y deforestadas (Los Limones).

El rango de hogar para fines de vegetación, ilustrado en la figura 6 , para todo el parque fue de 34.99 ha o $0.3 \mathrm{~km}$, con contrastes significativos entre las zonas: Sabana de la Mar posee 3 veces el valor de rango de la zona de Los Limones y 6 veces el rango de la zona de Cristal, como se puede observar en la figura.

La métrica distancia del vecino más cercano entre nidos (MEAN_ENN) presenta valores inferiores en el espaciamiento de anidación cuando se comparan sus cifras con los registros Dare y Barry (1990) para el Buteo buteo con 1950 \pm 750 m y Virani (1999) para el Buteo augur con 


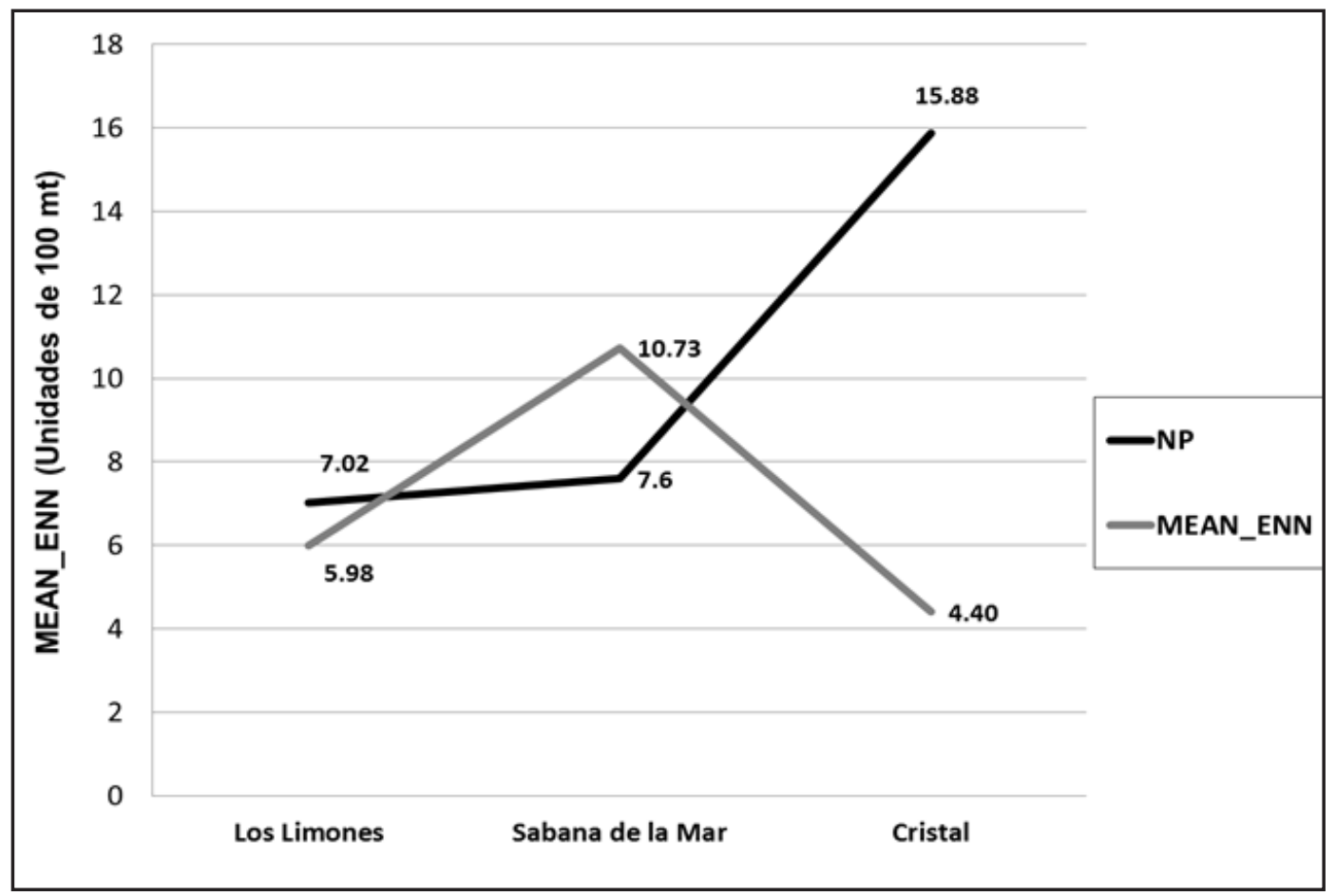

Figura 5: Valor MEAN_ENN y número de parches según zona para lugares de anidación del Buteo ridgwayi, Parque Nacional Los Haitises, República Dominicana (Elaboración propia).

$1350 \pm 90 \mathrm{~m}$. Con relación a estudios previos del Buteo ridgwayi, Wiley y Wiley (1981) reportó para el parque un valor MEAN_ENN de $727 \mathrm{~m}$ y un rango de hábitat de 57.8 ha, con un $\mathrm{n}$ de 3 nidos. Es decir, que en 39 años de diferencia entre los datos del Buteo ridgwayi y para un $n$ sumamente pequeño, el valor de MEAN_ENN se redujo en un $17 \%$; mientras que el rango de hábitat se redujo en 22.81 ha. En el caso del estudio de Woolaver (2011) que se localiza únicamente en la zona de Los Limones durante el período 2005-2009, el valor MEAN_ENN fue de $607 \pm 276$, que representa una diferencia de $8.52 \mathrm{~m}$ respecto al valor registrado para este estudio; la media de rango de hábitat reportada por Woolaver es de 32.58 ha, que indica muy poca variación entre las dos cifras.

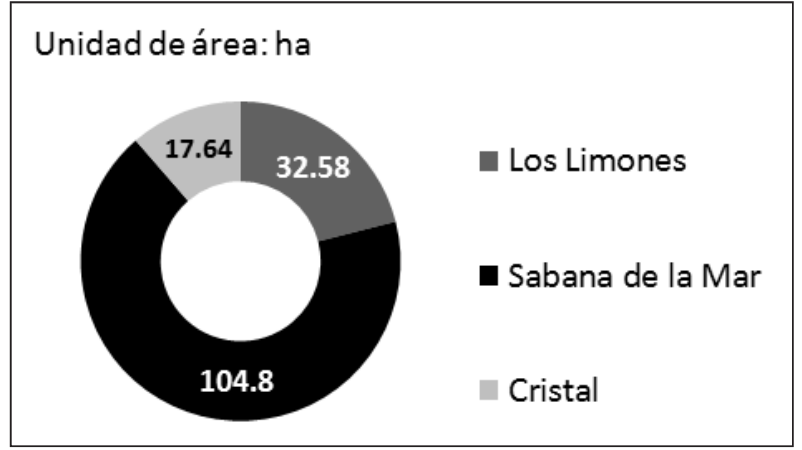

Figura 6: Rango de hogar en tres zonas de monitoreo de anidación del Buteo ridgwayi en el Parque Nacional Los Haitises, República Dominicana. (Elaboración propia) 
Cuadro 9

Historial de presencia del Buteo ridgwayi durante el período 2005 - 2015, Parque Nacional Los Haitises, República Dominicana (Elaboración propia).

\begin{tabular}{llll}
\hline Zona & Territorio & Total & Promedio \\
\hline Los Limones & La Cacata & 155 & 14.09 \\
& Los Arroyitos & 147 & 23.36 \\
& Mata de Limón & 114 & 10.36 \\
& Los Aguacaticos & 102 & 9.27 \\
& La Sierra & 61 & 5.55 \\
Trepada Alta & 47 & 11.75 \\
Sabana de la Mar & Llanada Grande & 20 & 5 \\
& Cano Hondo & 16 & 4 \\
Arroyón & 13 & 3.25 \\
& Monte Bonito & 12 & 3 \\
& El Caimoni & 49 & 12.25 \\
& Loma del Capa & 5 & 1.25 \\
& Los Britos & 21 & 5.25 \\
& Manantial del Queso & 25 & 6.25 \\
& Puerto Escondido & 5 & 1.25 \\
\hline
\end{tabular}

Cuadro 10

Métricas de paisaje para los lugares reocupados por el Buteo ridgwayi para el período 2005 - 2015 en el Parque Nacional Los Haitises, República Dominicana (Elaboración propia).

\begin{tabular}{lccccc}
\hline Variable & Unidad & Media & D.E. & Min & Max \\
\hline NP & $\%$ & 11.39 & 5.56 & 3 & 24 \\
LPI & 44.99 & 16.07 & 16.2 & 89.77 \\
ENN & $\mathrm{m}$ & 459 & 339.57 & 16 & 1803 \\
Home Range & ha & 33.52 & 54.03 & 0.02 & 296.33 \\
$\begin{array}{l}\text { Uso de suelo } \\
\text { (Matorral) }\end{array}$ & $\%$ & $44 \%$ & & &
\end{tabular}




\subsection{Identificación de áreas de reocupación}

Se encontraron 76 áreas registradas en todo el historial de reportes de presencia de nidos o avistamiento del Buteo ridgwayi en el Parque Nacional Los Haitises: 38 en Los Limones, 24 en Sabana de la Mar y 14 en Cristal. Para cada zona, se eligieron los cinco sitios con mayores registros de presencia a través del historial de monitoreo, que se detallan en el cuadro 9.

Respecto al árbol de anidación, solo se contaron con registros para los años 2008 - 2009, 2012 y 2014, donde en dichos períodos la Roystonea borinquena fue la especie de árbol con mayores registros como árbol de anidación. Para las métricas de paisaje, se consideraron los cinco lugares más revisitados por el Buteo ridgwayi en cada una de las tres zonas, para un total de 15 lugares en todo el parque y dentro de estos se examinaron 66 registros. En términos generales, las medias de cada variable se aproximaron a las medias obtenidas en 2015 según se muestra en el cuadro 10.

\section{Conclusiones}

Los patrones de uso de hábitat de la población monitoreada del Buteo ridgwayi en el Parque Nacional Los Haitises, revelados en esta investigación, confirmaron la preferencia documentada en su historial de monitoreo por la especie Roystonea borinquena como árbol de anidación. El uso de suelo matorral, con el mayor número de casos de anidación, posee la presencia de esta palma endémica debido a factores locales ligados a la tradición de la comunidad de Los Haitises y presencia de otras aves endémicas.

Para efectos de anidación, el Buteo ridgwayi tuvo presencia en diferentes usos de suelo que contaran con un árbol de anidación sobresaliente por sus características de altura y mor- fología, aún a pesar de encontrarse en condiciones de fragmentación y deforestación reveladas en las métricas de paisaje de las zonas de anidación. De esta forma, la población monitoreada del Buteo ridgwayi no muestra una dependencia de la cobertura bosque para efectos de anidación al estar anidando en áreas deforestadas. Con este estudio se demuestra que para fines de anidación de la población monitoreada del Buteo ridgwayi en zonas degradadas, la deforestación y la fragmentación no fueron factores limitantes; sin embargo, la presencia del Buteo ridgwayi en Los Haitises como único refugio natural bajo las características de ser bosque muy húmedo subtropical a menos de 1000 msnm, requiere aun de estudios que puedan establecer con mayor claridad la conexión de esta rapaz endémica con ese bosque, en términos de requerimientos y usos, debido a que su población está presente únicamente en esta zona.

\section{Recomendaciones}

Para los fines de un plan de conservación del Buteo ridgwayi que cuente con más criterios de comprensión sobre su proceso de extinción y requerimientos de hábitat, se recomienda profundizar en los siguientes aspectos:

\section{Zonas de entorno para protección de nidos del Buteo ridgwayi.}

Con la identificación del rango de hábitat y la distancia mínima al nido más cercano, como cifras que expresan la limitación de espaciamiento para distribución de los nidos en la población monitoreada del Buteo ridgwayi, se recomienda el establecimiento de zonas de entorno para evitar disturbios en los nidos de la especie en las actuales condiciones de fragmentación del parque, en un proceso de contacto y sensibilización con la población campesina que allí habita bajo usos contrarios al propósito de esta área protegida. 


\section{Importancia de la Roystonea borinquena.}

También se recomienda sensibilizar a la comunidad campesina con una actividad presente en la zona sobre la importancia de la especie Roystonea borinquena para la supervivencia del Buteo ridgwayi. También se debe reforzar el aprecio desde el punto de vista social de esta especie de flora a través de un proceso de diálogo y acercamiento con la población campesina mediante campañas de educación ambiental acerca de la condición del parque y la presencia del Buteo ridgwayi.

\section{Investigación de seguimiento sobre uso de hábitat del Buteo ridgwayi.}

A pesar de que, para fines de anidación, los resultados de esta investigación no revelaron una dependencia directa del Buteo ridgwayi con el uso de suelo bosque, la población del Buteo ridgwayi se redujo de una amplia distribución en todo el territorio dominicano a su única ubicación actual en Los Haitises. Se recomienda la realización de estudios acerca de la dieta del $\mathrm{Bu}-$ teo ridgwayi, para establecer su procedencia y posible conexión con el bosque de Los Haitises. De igual manera, se requiere estudiar los movimientos del gavilán dentro de la zona del parque para establecer el uso de rango de hábitat en una extensión mayor a sus actividades de anidación.

\section{Caracterizar lugares de antigua distri- bución del Buteo ridgwayi en el territorio dominicano.}

Como continuidad a la construcción de la historia natural de la especie y paralelamente a la caracterización de la zona de presencia actual, la realización de estudios que puedan dar información sobre las características ambientales de los antiguos lugares de anidación, permitiría buscar diferencias respecto a las caracte- rísticas de Los Haitises, para comprender qué hace de este lugar su refugio único en la actualidad y arrojar luz sobre su proceso de extinción.

5. Integración de resultados sobre la ecología de la larva Philornis pici a los estudios de hábitat del Buteo ridgwayi.

Desde el 2015, el equipo de The Peregrin fund realiza esfuerzos de investigación en la comprensión de la ecología de la larva de mosca Philornis pici para la evaluación del impacto de este parásito a la población del Buteo ridgwayi. Teniendo en cuenta de que el crecimiento de la población de esta larva de mosca ha sido asociado al aumento de las temperaturas en zonas naturales por efectos de deforestación, la integración de estos resultados a los estudios del hábitat del Buteo ridgwayi aportaría una visión más completa de efectos indirectos de la deforestación sobre su población.

\section{Referencias}

Andren, H. (1994). Effects of Habitat Fragmentation on Birds and Mammals in Landscapes with DifferentProportions of Suitable Habitat: A Review. [ Efectos de la fragmentación del hábitat en aves y mamíferos en el paisaje con diferentes proporciones de hábitat adecuado] Oikos (3).

Bielanski, W. (2006). Nesting preferences of common buzzard Buteo buteo and goshawk Accipiter gentilis in forest stands of different structure (Niepolomice Forest, Southern Poland). [Preferencias de anidación del ratonero común $\mathrm{Bu}-$ teo buteo y el azor Accipiter gentilis en bosques de diferentes estructuras]. Biología, Bratislava (5): 597-603. 
Carodenuto, S.; Merger, E.; Essomba, E.; Panev, M.; Pistorious, T.; Amougou, J. (2015). A Methodological Framework for Assessing Agents, Proximate Drivers and Underlying Causes of Deforestation: Field Test Results from Southern Cameroon. [Marco metológico para evaluar agentes impulsores inmediatos y las causas subyacentes de la deforestación: resultados de las pruebas de campo del sur de Camerún]. Forests (6): $203-224$.

Chalfoun, A.M., T. (2007). Assessments of habitat preferences and quality depend on spatial scale and metrics of fitness. [Las evaluaciones de las preferencias y calidad del hábitat dependen de la escala espacial y las mediciones de la aptitud física]. Journal of Applied Ecology. Disponible en http://www.umt. edu/mcwru/personnel/martin/PDF\%20 Martin/AppliedEcology_Chalfoun_ Martin07.pdf

Dalziel, B.D.; Morales, J.M.; Fryxell, J.M. (2008). Fitting probability distributions to animal movement trajectories: using artificial neural networks to link distance, resources, and memory. [Ajuste de las distribuciones de probabilidad a las trayectorias del movimiento de los animales: usando redes artificiales para vincular la distancia, los recursos y la memoria]. Am. Nat (248-258).

Dare, P.; Barry, J. (1990). Population size, density and regularity in nest spacing of Buzzards Buteo buteo in two upland regions of North Wales. [Tamaño de la población, densidad y regularidad en el espaciamiento de nidos de Buzzards Buteo buteo en dos regiones de tierras altas del norte de Gales]. Bird Study (1): 23-29.
Dec. No. 81-93. ( 1993). Que amplía la extensión territorial del Parque Nacional Los Haitises. Gaceta Oficial 9855 del 24 de marzo de 1993. República Dominicana. 1993.

Finegan, B. ( 2008). Capítulo 6: Patrones de fragmentación de los bosques de tierras bajas, su impacto en las comunidades y especies vegetales y propuestas para su mitigación. Santo Domingo, Costa Rica, INBIO. (Evaluación y conservación de biodiversidad en paisajes fragmentados de Mesoamérica)

Inselman, W.; Datta, S.; Jenks, J.; Jensen, K.; Grovenburg, T. (2015). Buteo Nesting Ecology: Evaluating Nesting of Swainson's Hawks in the Northern Great Plains. [Ecología de nidificación del anidado: evaluación de anidación de halcones de Swainson en las grandes llanuras del norte]. Plos One (9): 15. Disponible en http://journals.plos. org/plosone/article?id=10.1371/journal.pone.013704510.1371/journal. pone. 0137045

International, B. (2011). Species Factsheet: Buteo ridgwayi. [ Ficha de las especies: buteo ridgwayi] Disponible en http:// www.birdlife.org/datazone/speciesfactsheet.php? $\mathrm{id}=3501$

Kruger, O. (2002). Analysis of nest occupancy and nest reproduction in two sympatric raptors: common buzzard Buteo buteo and goshawk Accipiter gentilis. [Análisis de ocupación y reproducción de nidos en dos aves rapaces simpátricas: ratonero común Buteo buteo y azor Accipiter gentilis] ECOGRAPHY (523532). (The Encyclopedia of Earth) 
Perdomo, L.; Arias, Y.; León, Y.; Wege, D. (2010) a. Do018. Los Haitises. Jaragua, G. ed. Santo Domingo, República Dominicana, Grupo Jaragua, Programa IBA - Caribe Bird International. 6 p. (Áreas Importantes para la Conservación de las Aves en República Dominicana) Disponible en http://www.grupojaragua.org. do/documents/AICAS/DR-IBAS_2010 DO018_Los_Haitises.pdf

Rolstad, J. (1991). Consequences of forest fragmentation for the dynamics of bird populations: conceptual issues and the evidence. [Consecuencias de la deforestación para la dinámica de poblaciones de aves: problemas conceptuales y evidencia]. Biological Journal of the Linnean Society (1-2): 149-163.

Sodhi, N.; Posa, M.; Lee, T.; Warketing, I. (2008). Effects of Disturbance or Loss of Tropical Rainforest on Birds. [ Efectos de la perturbación o escasa lluvia tropical en las aves]. The Auk - An International Journal of Ornithology (3): 9.

The Peregrine Fund (TPF). (2012). Restoration of the Distribution and Abundance of Ridgway's Hawk in Historic Áreas Outside of Los Haitises National Park, Dominican Republic: Report Ridgway's Hawk Project 2012. [ Restauración de la distribución y abundacia del halcón de Ridgwayi en áreas históricas fuera del Parque Nacional de los Haitises, República Dominicana: informe del proyecto Hawk Ridgwayi 2012]. Fund, T.P. ed. Boyce, Idaho, Estados Unidos, The Peregrine Fund.

Throstrom, R.A., J.; Balbuena, S.; Rodríguez, P.F., E. (2005). Surveys and Breeding Biology of Buteo ridgwayi (Ridgway's hawk) in Los Haitises. [Encuestas y biología reproductiva del Buteo ridgwayi ( Ridgway's hawk) en los Haitises]. Caribbean Journal of Science (4): 864-869.

Turner, M. (1989). Landscape Ecology: The Effect of Pattern on Process. [ Ecología del paisaje: el efecto del patrón en el proceso]. Annu. Rev. Ecol. Syst. (171-97).

UICN. (2010). Buteo ridgwayi. In IUCN 2011. IUCN Red List of Threatened Species. Version 2011.1. International Union for the Conservation of Nature (IUCN). [Lista roja de especies amenazadas de la Unión Internacional lde Conservación de la Naturaleza]. Disponible en http://www.iucnredlist.org/

Urban, D.; O’Neill, R.; Shugart, R. (1987).

Landscape Ecology. A hierarchical perspective can help scientists understand spatial patterns. [Ecología del paisaje. Una perspectiva jerárquica puede ayudar a los científicos a comprender los patrones espaciales] BioScience: 119-127. Disponible en http://deathstar. rutgers.edu/people/mingxu/teaching/ landscape $\% 20$ ecology06/readings/urban87.PDF 
Wiley, J.; Wiley, B.(1981). Breeding season ecology and behaviour of Ridgway's Hawk Buteo ridgwayi. [Ecología y comportamiento de la temporada de cría del halcón de Ridgwai Buteo ridgwayi]. Condor - The Cooper Ornithological Society (83): $132-151$.
Zuidemaa, P.; Sayera, P.; Dijkmana, W. (1996). Forest fragmentation and biodiversity: the case for intermediate-sized conservation areas. [Fragmentación forestal y biodiversidad: el caso del área de conservación de tamaño intermedio]. Environmental Conservation (4): 290-297.

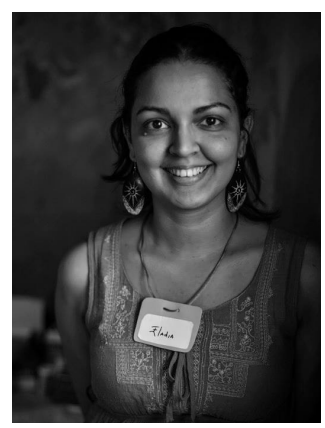

\section{Eladia Gesto De Jesús}

Licenciada en Antropología de la Universidad Autónoma de Santo Domingo, con Maestría Académica Internacional en Manejo y Conservación de Bosques Tropicales y Biodiversidad en el Centro Agronómico Tropical de Investigación y Enseñanza (CATIE), Costa Rica. Cuenta con una vasta experiencia en investigación contextual de la deforestación en áreas protegidas y programas de conservación para especies de fauna en extinción. Es además facilitadora de procesos de mediación entre el Ministerio de Medio Ambiente y comunidades campesinas respecto a temas de uso de tierras protegidas en nuestro país. Actualmente es la coordinadora de Proyectos de Conservación de la Fundación Propa-Gas. 